\title{
Intelligent biointerfaces applied to monitoring and homeodynamic communication between the hospital environment and the nursing staff body at CAISM-UNICAMP
}

\author{
Keywords \\ Architecture-Neurosciences, CAISM-UNICAMP, Homeodynamic Environments and Products, \\ Intelligent Biointerfaces, Pandemic, Post-pandemic Contexts.
}

The research aims to evidence the connections between of Architecture and Cognitive and Behavioral Neurosciences scientific fields, to analyze relationships between the hospital environment and the human body. The study focuses on the nursing professionals at the Center for Integral Care to Women (CAISM-UNICAMP), situated at University of Campinas campus at Campinas, Brazil and, in this context, articulates the production of somatosensory stimuli, the monitoring of vital signs and the monitoring of data from the hospital environment. So, it moves towards the integration and visualization of these data in a Building Information Modeling (BIM) platform. Therefore, the relationship between somatosensory stimuli, the performance of environmental factors and the homeodynamic balance of the body of these professionals is investigated in face of daily overloads - such physical or mental - in hospital environments, that became even more intensified by the impacts of the COVID-19 pandemic. The research is mainly supported by references available only in the English language, which reinforces its relevance for the national and international scenario of Portuguese language. The studies about Building Information Modeling advances in Eastman research on the Facility Manager (FM) stage, that is, maintenance of the building. Since the BIM model stores information from the hospital environment, it can also receive information from the human body of nursing professionals. Thus, the analytical data can be visualized on the platform, as BIM can communicate numerical and real-time data that can be viewed on an online platform. The research methodology comprises a transdisciplinary approach based on the concept of Homeodynamic Environments and Products and on the theoretical bases of Cognitive and Behavioral Neurosciences, especially from the perspective of the concept of biological homeostasis and homeodynamics, and Architecture with an emphasis on Building Information Modeling technology. In addition, this research is based on Design Science Research, and the creation of artifacts to build a device with the intention of solving a specific problem , it also involves field research aimed to the application of an experimental protocol, as well as the systematization, analysis, and interpretation of all collected data with a view to developing an integrated technological system oriented to the biological homeostasis of nursing professionals in COVID-19 pandemic and post-pandemic scenarios. Thus, the investigation is also practice-oriented, which allows the researcher to develop, apply and communicate original knowledge, in national and international contexts, with direct implications in the production of artifacts (software and hardware) and creation of new technological processes. 